\title{
Semiderivations Satisfying Certain Algebraic Identities on Jordan Ideals
}

\author{
Vincenzo De Filippis, ${ }^{1}$ Abdellah Mamouni, ${ }^{2}$ and Lahcen Oukhtite ${ }^{2}$ \\ ${ }^{1}$ Department of Mathematics and Computer Science, University of Messina, 98166 Messina, Italy \\ ${ }^{2}$ Université Moulay Ismail, Faculté des Sciences et Techniques, Département de Mathématiques, BP 509, \\ Boutalamine, 52000 Errachidia, Morocco \\ Correspondence should be addressed to Lahcen Oukhtite; oukhtitel@hotmail.com
}

Received 7 April 2013; Accepted 27 May 2013

Academic Editors: V. Drensky, K. Fujii, M. Ladra, J. van de Leur, A. Woldar, S. Yang, and Y. Zhou

Copyright (c) 2013 Vincenzo De Filippis et al. This is an open access article distributed under the Creative Commons Attribution License, which permits unrestricted use, distribution, and reproduction in any medium, provided the original work is properly cited.

\begin{abstract}
Let $R$ be a ring. An additive mapping $d: R \rightarrow R$ is called semiderivation of $R$ if there exists an endomorphism $g$ of $R$ such that $d(x y)=d(x) g(y)+x d(y)=d(x) y+g(x) d(y)$ and $d(g(x))=g(d(x))$, for all $x, y$ in $R$. Here we prove that if $R$ is a 2-torsion free *-prime ring and $J$ a nonzero *-Jordan ideal of $R$ such that $d([x, y])-[x, y]=0$ for all $x, y \in J$, then either $R$ is commutative or $d(x)=x-g(x)$ for all $x \in R$. Moreover, we initiate the study of generalized semiderivations in prime rings.
\end{abstract}

\section{Introduction and Preliminaries}

Throughout this paper, $R$ will denote an associative ring with center $Z(R)$. We will write, for all $x, y \in R,[x, y]=x y-y x$ and $x \circ y=x y+y x$ for the Lie product and Jordan product, respectively. $R$ is 2 -torsion free, if whenever $2 x=0$, with $x \in$ $R$, then $x=0 . R$ is prime if $a R b=0$ implies that $a=0$ or $b=0$. If $R$ admits an involution $*$, then $R$ is $*$-prime if $a R b=a R b^{*}=0$ yields $a=0$ or $b=0$. Note that every prime ring having an involution $*$ is $*$-prime, but the converse is in general not true. Indeed, if $R^{o}$ denotes the opposite ring of a prime ring $R$, then $R \times R^{o}$ equipped with the exchange involution $*_{\mathrm{ex}}$, defined by $*_{\mathrm{ex}}(x, y)=(y, x)$, is $*_{\mathrm{ex}}$-prime but not prime. This example shows that every prime ring can be injected in a $*$-prime ring, and from this point of view $*$ prime rings constitute a more general class of prime rings.

An additive mapping $d: R \rightarrow R$ is a derivation on $R$ if $d(x y)=d(x) y+x d(y)$ for all $x, y \in R$. Let $a \in R$ be a fixed element. A map $d: R \rightarrow R$ defined by $d(x)=[a, x]=$ $a x-x a, x \in R$, is a derivation on $R$, which is called inner derivation defined by $a$. Many results in the literature indicate how the global structure of a ring $R$ is often tightly connected to the behaviour of additive mappings defined on $R$. A wellknown result of Posner [1] states that if $d$ is a derivation of the prime ring $R$ such that $[d(x), x] \in Z(R)$, for any $x \in R$, then either $d=0$ or $R$ is commutative. In [2], Lanski generalizes the result of Posner to a Lie ideal.

More recently, several authors consider similar situation in the case that the derivation $d$ is replaced by a generalized derivation. More specifically, an additive map $G: R \rightarrow R$ is said to be a generalized derivation if there exists a derivation $d$ of $R$ such that, for all $x, y \in R, G(x y)=G(x) y+x d(y)$. Basic examples of generalized derivations are the usual derivations on $R$ and left $R$-module mappings from $R$ into itself. An important example is a map of the form $G(x)=a x+x b$, for some $a, b \in R$; such generalized derivations are called inner. Generalized derivations have been primarily studied on operator algebras. Therefore, any investigation from the algebraic point of view might be interesting (see, e.g., $[3,4]$ ).

In [5] Bergen introduced the notion of a semiderivation of a ring $R$ as follows: an additive mapping $d$ of $R$ into itself is called a semiderivation if there exists a function $g$ : $R \rightarrow R$ such that $d(x y)=d(x) g(y)+x d(y)=d(x) y+$ $g(x) d(y), d(g(x))=g(d(x))$ for all $x, y$ in $R$. In case $g$ is the identity map on $R, d$ is a derivation. Moreover, if $g$ is an automorphism of $R, d$ is called skew derivation (or $g$ derivation). Basic examples of $g$-derivations are the usual derivations and the map $g-1$, where 1 denotes the identity map. Let $b \in R$ be a fixed element. Then a map $D: R \rightarrow R$ 
defined by $D(x)=b x-g(x) b, x \in R$, is a $g$-derivation on $R$, and it is called an inner $g$-derivation (an inner skew derivation) defined by $b$.

An additive subgroup $J$ of $R$ is said to be a Jordan ideal of $R$ if $u \circ r \in J$, for all $u \in J$ and $r \in R$. A Jordan ideal $J$ which satisfies $J^{*}=J$ is called a $*$-Jordan ideal. We use without explicit mention the fact that if $J$ is a nonzero Jordan ideal of a ring $R$, then $2[R, R] J \subseteq J$ and $2 J[R, R] \subseteq J[6$, Lemma 2.4]. Moreover, from [7, proof of Lemma 3], we have $4 j^{2} R \subset J$ and $4 R j^{2} \subset J$ for all $j \in J$. Since $4 j r j=2\{j(j r+r j)+(j r+r j) j\}-$ $\left\{2 j^{2} \cdot r+r \cdot 2 j^{2}\right\}$, it follows that $4 j R j \subset J$ for all $j \in J$ (see [7, proof of Theorem 3]).

Recently, many authors have studied commutativity of prime and semiprime rings admitting suitably constrained additive mappings, as automorphisms, derivations, skew derivations, and generalized derivations acting on appropriate subsets of the rings. In the present paper, we would like to study the structure of a $*$-prime ring having a semiderivation which satisfies suitable algebraic properties on *-Jordan ideals of $R$. More precisely, we will prove the following.

Theorem 1. Let $R$ be a 2-torsion free *-prime ring, and let $J$ be a nonzero *-Jordan ideal of $R$. If $R$ admits a nonzero semiderivation $d$ (with associated endomorphism $g$ ) such that $d([x, y])-[x, y]=0$ for all $x, y \in J$, then $R$ is commutative or $d(x)=x-g(x)$ for all $x \in R$.

Motivated by the concept of semiderivation, in Section 3, we introduce the concept of generalized semiderivation.

Definition 2. Let $R$ be a ring and $F: R \rightarrow R$ an additive map. If there is a semiderivation $d: R \rightarrow R$ associated with the function $g: R \rightarrow R$ such that

$$
\begin{gathered}
F(x y)=F(x) y+g(x) d(y)=d(x) g(y)+x F(y), \\
F(g(x))=g(F(x))
\end{gathered}
$$

for each $x, y \in R$, then $F$ is called a generalized semiderivation of $R$, associated with the function $g$ and the semiderivation $d$.

Of course any semiderivation is a generalized semiderivation. Moreover, if $g$ is the identity map of $R$, then all generalized semiderivations associated with $g$ are merely generalized derivations of $R$. Furthermore, we have already seen that if $R$ is prime and $d \neq 0$, then $g$ must be a ring endomorphism.

Example 3. Let $R$ be a ring and $d: R \rightarrow R$ a semiderivation of $R$ associated with a function $g$ of $R$. Define $F: R \rightarrow R$ and $G: R \rightarrow R$ as follows:

$$
F(x)=d(x)-x, \quad G(x)=d(x)+x, \quad \forall x \in R .
$$

It is easy to check that $F$ and $G$ satisfy (1), so that $F$ and $G$ are generalized semiderivations of $R$ associated with $g$.

The definition of generalized semiderivations unifies the notions of semiderivation and generalized derivation and covers the concepts of derivations, generalized derivations, left (right) centralizers, and semiderivations. Thus, in the last part of this note, we give a characterization of generalized semiderivations in prime rings and show that any generalized semiderivation of a prime ring $R$ assumes essentially only two possible forms.

\section{Commutativity Conditions on Semiderivations}

Throughout, $R$ will be a 2 -torsion free $*$-ring, $J$ a nonzero $*$ Jordan ideal of $R$, and $S a_{*}(R):=\left\{r \in R \mid r^{*}= \pm r\right\}$ the set of symmetric and skew symmetric elements.

We make some use of the following well-known results.

Remark 4. Let $R$ be a 2 -torsion free $*$-prime $\operatorname{ring}$ and $J$ a nonzero $*$-Jordan ideal.

(1) If $a J b=a^{*} J b=0$, then $a=0$ or $b=0$ ([8, Lemma 2]).

(2) If $[J, J]=0$, then $R$ is commutative ([9, Lemma 2.5]).

(3) If $\left[x, y^{2}\right]=0$ for all $x, y \in J$, then $R$ is commutative ([9, proof of Theorem 3]).

We leave the proofs of the following two facts to the reader.

(4) If $a J a=0$, then $a=0$. In particular, if $a J=0$ or $J a=0$, then $a=0$.

(5) If $r \in R$ is such that $[[s, t], r]=0$ for all $s, t \in R$, then $r \in Z(R)$.

Lemma 5. Let $R$ be a 2-torsion free *-prime ring and $J a$ nonzero $*$-Jordan ideal of $R$. If $a \in R$ is such that $\left[a,\left[x, y^{2}\right]\right]=$ 0 for all $x, y \in J$, then $a \in Z(R)$.

Proof. Assume that

$$
\left[a,\left[x, y^{2}\right]\right]=0, \quad \forall x, y \in J .
$$

Replacing $x$ by $4 x y^{2}$ in (3), we get

$$
\left[x, y^{2}\right]\left[a, y^{2}\right]=0, \quad \forall x, y \in J .
$$

Substituting $2[r, s] x$ for $x$ in (4), where $r, s \in R$, we obtain $\left[[r, s], y^{2}\right] x\left[a, y^{2}\right]=0$ so that

$$
\left[[r, s], y^{2}\right] J\left[a, y^{2}\right]=0, \quad \forall y \in J, r, s \in R .
$$

For $y \in S a_{*}(R) \cap J$, either $\left[a, y^{2}\right]=0$ or $\left[[r, s], y^{2}\right]=0$, in which case Remark 4(5) forces $y^{2} \in Z(R)$. Hence, in both cases, we have $\left[a, y^{2}\right]=0$.

Let $y \in J$, as $y-y^{*}, y+y^{*} \in S a_{*}(R) \cap J$; then $\left[a,\left(y-y^{*}\right)^{2}\right]=\left[a,\left(y+y^{*}\right)^{2}\right]=0$ so that $\left[a, y^{2}\right]=-\left[a,\left(y^{*}\right)^{2}\right]$; replacing $y$ by $y^{*}$ in (5), we find that

$$
\left(\left[[r, s], y^{2}\right]\right)^{*} J\left[a, y^{2}\right]=0, \quad \forall y \in J, r, s \in R .
$$

Combining (5) with (6), we arrive at $\left[a, y^{2}\right]=0$ which by linearization yields

$$
[a, x \circ y]=0, \quad \forall x, y \in J .
$$


Writing $4 y z^{2}$ instead of $y$ in (7), where $z \in J$, we get

$$
[a, y]\left[x, z^{2}\right]=0 \quad \forall x, y, z \in J .
$$

Substituting $2[r, s] y$ for $y$ in (8), where $r, s \in R$, we obtain $[a,[r, s]] y\left[x, z^{2}\right]=0$ and thus

$$
[a,[r, s]] J\left[x, z^{2}\right]=0, \quad \forall x, z \in J, r, s \in R .
$$

Since $J$ is invariant under $*$, because of (9), in view of Remark 4(1) either $[a,[r, s]]=0$ for all $r, s \in R$ so, by Remark 4(5), $a \in Z(R)$ or $\left[x, z^{2}\right]=0$. According to Remark 4(3), the last case assures that $R$ is commutative and therefore $a \in Z(R)$.

Lemma 6. Let $R$ be a 2-torsion free *-prime ring and $J a$ nonzero *-Jordan ideal of $R$. If $R$ admits a semiderivation $d$ such that $d\left(x^{2}\right)=0$ for all $x \in J$, then $d=0$.

Proof. Assume that

$$
d\left(x^{2}\right)=0, \quad \forall x \in J
$$

Linearizing (10), we obtain

$$
d(x \circ y)=0, \quad \forall x, y \in J .
$$

Replacing $y$ by $4 z^{2} y^{2}$ in (11), where $z \in J$, we get

$$
z^{2} d\left(\left[x, y^{2}\right]\right)=0, \quad \forall x, y, z \in J .
$$

Substituting $z+u$ for $z$, we find that $(z \circ u) d\left(\left[x, y^{2}\right]\right)=0$, and replacing $u$ by $2[r, s] u$ in the last expression, where $r, s \in R$, we arrive at

$$
\begin{array}{r}
{[z,[r, s]] J d\left(\left[x, y^{2}\right]\right)=([z,[r, s]])^{*} J d\left(\left[x, y^{2}\right]\right)=0,} \\
\forall x, y, z \in J, \quad r, s \in R
\end{array}
$$

In view of Remark 4(1), from (13), it follows that either $d\left(\left[x, y^{2}\right]\right)=0$ or $[z,[r, s]]=0$ for all $z \in J, r, s \in R$, in which case Remark 4(5) forces $J \subseteq Z(R)$ and [10, Lemma 3] implies that $R$ is commutative. Accordingly, in both cases, we find that

$$
d\left(\left[x, y^{2}\right]\right)=0, \quad \forall x, y \in J
$$

and therefore

$$
\left[d(x), y^{2}\right]=0, \quad \forall x, y \in J
$$

Linearizing (15), we obtain

$$
[d(x), y \circ z]=0, \quad \forall x, y, z \in J .
$$

Replacing $z$ by $4 z u^{2}$ in (16), where $u \in J$, we get

$$
\left[d(x),(y \circ z) u^{2}-z\left[y, u^{2}\right]\right]=0, \quad \forall u, x, y, z \in J .
$$

Therefore

$$
z\left[d(x),\left[y, u^{2}\right]\right]+[d(x), z]\left[y, u^{2}\right]=0, \quad \forall u, x, y, z \in J .
$$

Substituting $2[r, s] z$ for $z$ in (18), where $r, s \in R$, we obtain $[d(x),[r, s]] z\left[y, u^{2}\right]=0$ thereby

$$
\begin{array}{r}
{[d(x),[r, s]] J\left[y, u^{2}\right]=[d(x),[r, s]] J\left(\left[y, u^{2}\right]\right)^{*}=0,} \\
\forall u, x, y \in J, \quad r, s \in R .
\end{array}
$$

In light of Remark 4(1), (19) implies that $[d(x),[r, s]]=$ 0 or $\left[y, u^{2}\right]=0$. Applying Remark 4(3) together with Remark 4(5), we conclude that $d(x) \in Z(R)$ for all $x \in J$. Accordingly,

$$
[d(x), r]=0, \quad \forall x \in J, r \in R .
$$

Replacing $x$ by $4 x^{2} y$ in (20), we get

$$
\left[x^{2}, r\right] d(y)=0, \quad \forall x, y \in J, r \in R \text {. }
$$

Substituting $r t$ for $r$ in (21) we get $\left[x^{2}, r\right] t d(y)=0$ so that

$$
\left[x^{2}, r\right] R d(y)=\left(\left[x^{2}, r\right]\right)^{*} R d(y)=0, \quad \forall x, y \in J, r \in R .
$$

Using the $*$-primeness hypothesis together with (22), we get $\left[x^{2}, r\right]=0$ or $d(J)=\{0\}$.

Assume that $d(J)=\{0\}$; from $d(r \circ j)=0$, it follows that

$$
j d(r)+d(r) j=0, \quad \forall j \in J, r \in R .
$$

Replacing $r$ by $r x$ in (23), with $x \in J$, we get

$$
d(r)[x, j]=0, \quad \forall j, x \in J, r \in R .
$$

Substituting $r y$ for $r$ in (23), with $y \in J$, we obtain $d(r) y[x, j]=0$ so that

$$
d(r) J[x, j]=0 \quad \forall j, x \in J, r \in R .
$$

In light of Remark 4(1), because of $J^{*}=J,(25)$ yields $d=0$ or $[J, J]=\{0\}$ in which case $R$ is commutative by Remark 4(2). Hence (23) together with 2-torsion freeness forces $d(r) J=\{0\}$ and Remark 4(4) assures that $d=0$.

If $\left[x^{2}, R\right]=\{0\}$ for all $x \in J$, then $R$ is commutative by Remark 4(3), in which case (10) becomes $d(x) R x=0$. Hence $d(x)=0$ for all $x \in J$ and thus $d=0$.

Proposition 7. Let $R$ be a 2-torsion free *-prime ring and $J$ a nonzero *-Jordan ideal of $R$. If $R$ admits a nonzero semiderivation $d$ such that $d([x, y])=0$ for all $x, y \in J$, then $R$ is commutative.

Proof. Assume that

$$
d([x, y])=0, \quad \forall x, y \in J
$$

Suppose that $Z(R) \cap J=0$; replacing $x$ by $4 x y^{2}$ in (26), we find that

$$
[x, y] d\left(y^{2}\right)=0 \quad \forall x, y \in J
$$


Substituting $2[r, s] x$ for $x$ in (27), where $r, s \in R$, we obtain $[[r, s], y] x d\left(y^{2}\right)=0$ in such a way that

$$
[[r, s], y] J d\left(y^{2}\right)=0, \quad \forall y \in J, r, s \in R .
$$

Using Remark 4(1) together with (28), we conclude that

$$
[[r, s], y]=0 \quad \text { or } \quad d\left(y^{2}\right)=0, \quad \forall y \in J \cap S a_{*}(R) .
$$

As $y-y^{*}, y-y^{*} \in J \cap S a_{*}(R)$ for all $y \in J$, one can easily see that

$$
[[r, s], y]=0 \quad \text { or } \quad d\left(y^{2}\right)=0, \quad \forall y \in J .
$$

If $[[r, s], y]=0$ for all $r, s \in R$, then $y \in Z(R)$ by Remark 4(5) which, because of $Z(R) \cap J=0$, forces $y=0$. Hence, in both cases, we arrive at

$$
d\left(y^{2}\right)=0, \quad \forall y \in J
$$

and Lemma 6 yields $d=0$ which contradicts our hypothesis. Hence

$$
J \cap Z(R) \neq 0 .
$$

Let $0 \neq z \in J \cap Z(R)$; replacing $x$ by $2 x z=x \circ z$ in (26), we obtain

$$
[x, y] d(z)=0, \quad \forall x, y \in J .
$$

Writing $2[r, s] x$ instead of $x$ in (33), where $r, s \in R$, we get $[[r, s], y] x d(z)=0$ and thus

$$
\begin{array}{r}
{[[r, s], y] J d(z)=0=([[r, s], y])^{*} J d(z),} \\
\forall y \in J, \quad r, s \in R .
\end{array}
$$

In light of Remark 4(1), (34) forces $[[r, s], y]=0$ or $d(z)=$ 0 . If $[[r, s], J]=\{0\}$ for all $r, s \in R$, then Remark 4(5) implies that $J \subseteq Z(R)$ and [10, Lemma 3] implies that $R$ is commutative.

Assume that $d(z)=0$ for all $z \in J \cap Z(R)$; replacing $x$ by $2 r z=r \circ z$ in (26), where $r \in R$, we get

$$
d([r, y]) z=0, \quad \forall y \in J, r \in R .
$$

In view of Remark 4(4), the last equation forces

$$
d([r, y])=0 \quad \forall y \in J, r \in R
$$

Reasoning as previous mentioned, we arrive at

$$
d([r, s])=0 \quad \forall r, s \in R .
$$

Substituting $[r, t] u$ for $r$ in (37), we get

$$
[[r, t], s] d(u)=0, \quad \forall r, s, t, u \in R .
$$

Replacing $s$ by $s v$ in $(38)$, we obtain $[[r, t], s] v d(u)=0$ so that

$$
[[r, t], s] R d(u)=0, \quad \forall r, s, t, u \in R .
$$

Since $d$ is a nonzero semiderivation, then (39) forces $[[r, t], s]=0$ for all $r, s, t \in R$, and thus $R$ is commutative.
Bell and Daif [11, Theorem 3] showed that if a prime ring $R$ admits a nonzero derivation $d$ satisfying $d([x, y])=0$ for all $x, y$ in a nonzero ideal $I$ of $R$, then $R$ is commutative. Our aim in the following theorem is to generalize this result of Bell and Daif in two directions. First of all, we will only assume that the commutativity condition is imposed on a Jordan ideal of $R$ rather than on a two sided ideal. Secondly we will treat the case of semiderivation instead of derivation but only with further assumption that the ring $R$ is 2 -torsion free.

Corollary 8. Let $R$ be a 2-torsion free prime ring and $J a$ nonzero Jordan ideal of $R$. If $R$ admits a nonzero semiderivation $d$ such that $d([x, y])=0$ for all $x, y \in J$, then $R$ is commutative.

Proof. Assume that $d$ is a nonzero semiderivation of $R$ such that $d([x, y])=0$ for all $x, y \in J$. Let us consider $\mathscr{R}=R \times R^{0}$, and set

$$
\begin{array}{r}
\mathscr{D}(x, y)=(d(x), 0), \quad \mathscr{G}(x, y)=(g(x), g(y)), \\
\forall x, y \in R .
\end{array}
$$

Clearly, $\mathscr{D}$ is a nonzero semiderivation of $\mathscr{R}$ associated with $\mathscr{G}$. Moreover, if we set $\mathscr{J}=J \times J$, then $\mathscr{J}$ is a $*_{\text {ex }}$-Jordan ideal of $\mathscr{R}$ and $\mathscr{D}([x, y])=0$ for all $x, y \in \mathscr{J}$. Since $\mathscr{R}$ is $*_{\text {ex }}$-prime, in view of Theorem 1 , we deduce that $\mathscr{R}$ is commutative and a fortiori $R$ is commutative.

Now if we consider the particular case where $g$ is the identity map in Corollary 8, we obtain [9, Theorem 2.6].

Corollary 9 (see $[9$, Theorem 2.6]). Let $R$ be a 2-torsion free prime ring and $J$ a nonzero Jordan ideal of $R$. If $R$ admits a nonzero derivation $d$ such that $d([x, y])=0$ for all $x, y \in J$, then $R$ is commutative.

Theorem 10. Let $R$ be a 2-torsion free *-prime ring, and let $J$ be a nonzero *-Jordan ideal of $R$. If $R$ admits a nonzero semiderivation $d$ (with associated endomorphism $g$ ) such that $d([x, y])-[x, y]=0$ for all $x, y \in J$, then $R$ is commutative or $d(x)=x-g(x)$ for all $x \in R$.

Proof. Assume that

$$
d([x, y])-[x, y]=0, \quad \forall x, y \in J
$$

Then

$$
[d(x), y]+[g(x), d(y)]-[x, y]=0, \quad \forall x, y \in J
$$

Writing $2\left[u, v^{2}\right]$ instead of $y$ in (42), we get

$$
\left[d(x)+g(x)-x,\left[u, v^{2}\right]\right]=0, \quad \forall x, u, v \in J .
$$

Then by Lemma 5, we have $d(x)+g(x)-x \in Z(R)$, and replacing $y$ by $4 x y^{2}$ in $(41)$, we get $(d(x)+g(x)-x)\left[x, y^{2}\right]=0$ for all $x, y \in J$ so that

$$
(d(x)+g(x)-x) R\left[x, y^{2}\right]=0, \quad \forall x, y \in J .
$$


For $x \in S a_{*}(R) \cap J$, in light of $*$-primeness, (44) yields $d(x)+$ $g(x)-x=0$ or $\left[x, y^{2}\right]=0$ for all $y \in J$.

Let $x \in J$, as $x-x^{*} \in S a_{*}(R) \cap J$; then we have $d\left(x-x^{*}\right)+$ $g\left(x-x^{*}\right)-\left(x-x^{*}\right)=0$ or $\left[x-x^{*}, y^{2}\right]=0$ for all $y \in J$.

If $d\left(x-x^{*}\right)+g\left(x-x^{*}\right)-\left(x-x^{*}\right)=0$, then $d(x)+g(x)-x=$ $d\left(x^{*}\right)+g\left(x^{*}\right)-x^{*}$. Hence, replacing $x$ by $x^{*}$ in (44), we arrive at $(d(x)+g(x)-x) R\left(\left[x, y^{2}\right]\right)^{*}=0$ which combined with (44) forces $d(x)+g(x)-x=0$ or $\left[x, y^{2}\right]=0$.

If $\left[x-x^{*}, y^{2}\right]=0$, then $\left[x, y^{2}\right]=\left[x^{*}, y^{2}\right]$ for all $y \in J$, and thereby (44) yields

$$
(d(x)+g(x)-x) R\left(\left[x, y^{2}\right]\right)^{*}=0,
$$

and once again using (44), because of the $*$-primeness, the last equation implies that $d(x)+g(x)-x=0$ or $\left[x, y^{2}\right]=0$. Accordingly, in both cases, we conclude that for all $x, y \in J$ either $\left[x, y^{2}\right]=0$ or $d(x)+g(x)-x=0$. Hence $J$ is a union of two additive subgroups $J_{1}$ and $J_{2}$, where

$$
\begin{aligned}
& J_{1}=\left\{x \in J \mid\left[x, y^{2}\right]=0, \forall y \in J\right\}, \\
& J_{2}=\{x \in J \mid d(x)+g(x)-x=0\} .
\end{aligned}
$$

Since a group cannot be a union of two of its proper subgroups, we are forced to have $J=J_{1}$ or $J=J_{2}$. If $J=J_{1}$, then $R$ is commutative by Remark 4(3). Now assume that $J=J_{2}$; then

$$
d(x)+g(x)-x=0, \quad \forall x \in J
$$

Replacing $x$ by $4 x^{2} r$ in (47) where $r \in R$, we get

$$
x^{2}(d(r)+g(r)-r)=0, \quad \forall x \in J, r \in R,
$$

which forces

$$
(x \circ y)(d(r)+g(r)-r)=0, \quad \forall x, y \in J, r \in R .
$$

Replacing $y$ by $2[u, v] y$ in the last equation, we obtain $[x,[u, v]] y(d(r)+g(r)-r)=0$ so that

$$
[x,[u, v]] J(d(r)+g(r)-r)=0, \quad \forall x \in J, r, u, v \in R .
$$

Since $J$ is invariant under $*$, then $[x,[u, v]]^{*} J(d(r)+g(r)-r)=$ 0 which combined with (50) assures, because of Remark 4(1), that either $(d(r)+g(r)-r)=0$ or $[J,[u, v]]=\{0\}$, in which case we find that $R$ is commutative. In conclusion, $R$ is commutative or $d(x)=x-g(x)$ for all $x \in R$.

Corollary 11 (see [9, Theorem 2.12]). Let $R$ be a 2-torsion free *-prime ring and $J$ a nonzero *-Jordan ideal of $R$. If $R$ admits a nonzero derivation $d$ such that $d([x, y])=[x, y]$ for all $x, y \in$ $J$, then $R$ is commutative.

Proof. $d$ being a nonzero derivation implies that it is a semiderivation associated with the identity endomorphism of $R$. Applying Theorem 10, because of $1=g \neq 1-d$, then we conclude that $R$ is commutative.
In case $R$ is a prime ring and $d \neq 0$ is a semiderivation with associated function $g$, Chang ([12], Theorem 1) has shown that $g$ must necessarily be a ring endomorphism. Using this result and reasoning as in the proof of Corollary 8 , application of Theorem 10 yields the following result.

Theorem 12. Let $R$ be a 2-torsion free prime ring and $J a$ nonzero Jordan ideal of $R$. If $R$ admits a nonzero semiderivation $d$ with associated function $g$ such that $d([x, y])-[x, y]=0$ for all $x, y \in J$, then $R$ is commutative or $d(x)=x-g(x)$ for all $x \in R$.

Lemma 13. Let $R$ be a prime ring, I a nonzero ideal of $R$, and $d$ a nonzero semiderivation of $R$, with associated function $g$, such that $d(x)=x-g(x)$, for all $x \in R$.

$$
\text { If } g(x) a=0 \text { for all } x \in I \text {, then either } a=0 \text { or } g(I)=0 \text {. }
$$

Proof. For any $x \in I$, by our assumption, we have $d(x) a=x a$. Thus, for any $r \in R$, it follows that $d(x r) a=x r a$, that is, $d(x) g(r) a+x d(r) a=x r a$ which implies that $d(x) g(r) a=$ $x g(r) a$. Now we replace $r$ by $r y$, for any $y \in I$. Hence we get $d(x) g(r y) a=x g(r y) a$, and by the definition of $d$, it follows that

$$
\begin{aligned}
d(x) d(r) y a & +d(x) g(r) d(y) a \\
& =x d(r) y a+x g(r) d(y) a,
\end{aligned}
$$

and since $d(y) a=y a$, one has

$$
d(x) d(r) y a+d(x) g(r) y a=x d(r) y a+x g(r) y a
$$

that is,

$$
(d(x) d(r)+d(x) g(r)-x d(r)-x g(r)) I a=0 .
$$

By the primeness of $R$, we have that either $a=0$ or $d(x) d(r)+$ $d(x) g(r)-x d(r)-x g(r)=0$. In this last case, since $d(r)+$ $g(r)=r$, it follows that $(d(x)-x) r=0$ for any $x \in I$ and $r \in R$, implying that $d(x)=x$. Thus we get that $g(I)=0$.

Theorem 14. Let $R$ be a 2-torsion free prime ring and $I a$ nonzero ideal of $R$. If $R$ admits a nonzero semiderivation $d$ with associated function $g$ such that $d([x, y])-[x, y]=0$ for all $x, y \in I$, then one of the following holds:

(1) $R$ is commutative;

(2) $d(x)=x-g(x)$ for all $x \in R$, with $g([R, R])=0$;

(3) $d(x)=x$, for all $x \in I$ and $g(I)=0$.

Proof. Assume that $R$ is not commutative. Thus, by Theorem 12, we have $d(x)=x-g(x)$ for all $x \in R$; therefore by our assumption, $g([I, I])=0$.

In particular, for any $x, y \in I$ and $r \in R$ we have both

$$
\begin{gathered}
d([x, y] r)=[x, y] r-g([x, y] r), \\
d([x, y] r)=d([x, y]) r+g([x, y]) d(r)=d([x, y]) r,
\end{gathered}
$$


which implies that

$$
\begin{aligned}
0 & =(d([x, y])-[x, y]) r+g([x, y] r) \\
& =-g([x, y]) r+g([x, y] r)=g([x, y] r),
\end{aligned}
$$

that is, for any $x, y \in I$ and $r \in R$,

$$
d([x, y] r)=[x, y] r .
$$

On the other hand,

$$
\begin{aligned}
d([x, y] r) & =d([x, y r]-y[x, r]) \\
& =[x, y r]-d(y[x, r]) \\
& =y[x, r]+[x, y] r-d(y[x, r]) .
\end{aligned}
$$

Comparing (56) with (57), we get

$$
y[x, r]=d(y[x, r])=d(y)[x, r]+g(y) d([x, r]),
$$

that is,

$$
g(y) g([x, r])=0
$$

for all $x, y \in I, r \in R$. By applying Lemma 13, either $g(I)=0$ or $g([I, R])=0$.

Here we assume that $g(I) \neq 0$, and hence $g([I, R])=0$. In this case, for any $x \in I$ and $r, s \in R$, we have both

$$
\begin{gathered}
d([x, r] s)=[x, r] s-g([x, r] s), \\
d([x, r] s)=d([x, r]) s+g([x, r]) d(s)=d([x, r]) s
\end{gathered}
$$

which implies that

$$
\begin{aligned}
0 & =(d([x, r])-[x, r]) s+g([x, r] s) \\
& =-g([x, r]) s+g([x, r] s)=g([x, r] s),
\end{aligned}
$$

that is, for any $x \in I$ and $r, s \in R$,

$$
d([x, r] s)=[x, r] s .
$$

On the other hand,

$$
\begin{aligned}
d([x, r] s) & =d([x s, r]-x[s, r]) \\
& =[x s, r]-d(x[s, r]) \\
& =[x, r] s+x[s, r]-d(x[s, r]) .
\end{aligned}
$$

Comparing (62) with (63), we get

$$
x[s, r]=d(x[s, r])=d(x)[s, r]+g(x) d([s, r]),
$$

that is,

$$
g(x) g([s, r])=0,
$$

for all $x \in I, r, s \in R$. By applying again Lemma 13, and since $g(I) \neq 0$, it follows that $g([R, R])=0$.

The following example proves that Proposition 7 and Theorem 10 cannot be extended to semiprime rings.
Example 15. Let $(R, \sigma)$ be a noncommutative prime ring with involution. If we set $\mathscr{R}=R \times Q[X]$, then $\mathscr{R}$ is a semiprime ring and $(r, P(X))^{*}=(\sigma(r), P(X))$ is an involution of $\mathscr{R} . J=$ $\{0\} \times Q[X]$ is a nonzero $*$-Jordan ideal of $\mathscr{R} . D(r, P(X))=$ $\left(0, P^{\prime}(X)\right)$ is a nonzero semiderivation of $\mathscr{R}$ associated with identity. We have $[u, v]=0$ for all $u, v \in J$ so that

$$
D([u, v])=0, \quad D([u, v])=[u, v], \quad \forall u, v \in J,
$$

but $\mathscr{R}$ is a noncommutative ring.

The following example demonstrates that the hypothesis " $J$ a *-Jordan ideal" in Proposition 7 is crucial.

Example 16. Let $R$ be a noncommutative prime ring which admits a nonzero derivation $d$, and let $\mathscr{R}=R \times R^{0}$. If we set $J=R \times\{0\}$, then $J$ is a nonzero Jordan ideal of the $*_{\text {ex }}$-prime ring $\mathscr{R}$. Furthermore, if we define $D(x, y)=(0, d(y))$, then $D$ is a semiderivation of $\mathscr{R}$ associated with identity which satisfies

$$
D([u, v])=0, \quad \forall u, v \in J .
$$

However $\mathscr{R}$ is a noncommutative ring.

\section{Generalized Semiderivations in Prime Rings}

In this final section, we study generalized semiderivations in prime rings. Then we assume that $R$ is a prime ring and that $F: R \rightarrow R$ is an additive map such that

$$
\begin{gathered}
F(x y)=F(x) y+g(x) d(y)=d(x) g(y)+x F(y) \\
F(g(x))=g(F(x)),
\end{gathered}
$$

for each $x, y \in R$, where $d: R \rightarrow R$ and $g: R \rightarrow R$ are, respectively, a semiderivation and an endomorphism of $R$.

Our aim here is to show that any generalized semiderivation of a prime ring $R$ assumes essentially one of the following forms.

Theorem 17. Let $R$ be a prime ring and $F: R \rightarrow R$ a generalized semiderivation of $R$ associated with the endomorphism $g$ and semiderivation $d$ of $R$. Then either one of the following two cases holds.

(1) The endomorphism $g$ is the identity map of $R$ and $F$ is a generalized derivation of $R$.

(2) There exist $\alpha, \beta \in C$, the extended centroid of $R$, such that $F(x)=\alpha x+\beta g(x)=(\alpha+\beta) x+d(x)$, for all $x \in R$.

Proof. By the definition of generalized semiderivation, we have that, for all $x, y \in R$,

$$
F(x) y+g(x) d(y)=d(x) g(y)+x F(y) .
$$

Assume that $d$ is not an ordinary derivation of $R$.

In this case, it is known that there exists $\lambda \in C$ such that $d(x)=\lambda(x-g(x))$, for all $x \in R$. In light of this and by (69), one has

$$
\begin{aligned}
F(x) y & +\lambda g(x) y-\lambda g(x) g(y) \\
& =\lambda x g(y)-\lambda g(x) g(y)+x F(y)
\end{aligned}
$$


that is,

$$
F(x) y+\lambda g(x) y-\lambda x g(y)-x F(y)=0 .
$$

Denote $G=F+\lambda g$. If $G=0$, then $F(x)=-\lambda g(x)$, for all $x \in R$, and there is nothing to prove. Hence we assume that $G \neq 0$, and by (71), we get

$$
G(x) y-x G(y)=0, \quad \forall x, y \in R .
$$

In particular for $x=y$ in (72), it follows that $[G(x), x]=0$; that is, $G$ is an additive commuting map on $R$. By [13, Theorem 3.2], there exist $\alpha \in C$ and an additive mapping $\theta: R \rightarrow C$, such that $G(x)=\alpha x+\theta(x)$, for all $x \in R$. Thus (72) implies that

$$
\theta(x) y-\theta(y) x=0, \quad \forall x, y \in R
$$

In case $\theta(R) \neq 0$ and since $\theta(R) \subseteq C$, by (73), we have $[x, y]=$ 0 in $R$; that is, $R$ is commutative. Moreover, since $G \neq 0$, then there exists $x_{0} \in R$ such that $G\left(x_{0}\right) \neq 0$ and denote $G\left(x_{0}\right)=$ $\gamma \in C$, and $x_{0}^{-1}=\eta \in C$. Thus, by (72), we have that $\eta \gamma y=$ $G(y)$, for all $y \in R$, that is, $F(y)=(\eta \gamma) y-\lambda g(y)$, for all $y \in R$, as required.

Hence we may consider the case $\theta(R)=0$ and $G(x)=\alpha x$, that is, $F(x)=\alpha x-\lambda g(x)$, for all $x \in R$, and we are done again.

We finally consider the case that $d$ is an ordinary derivation of $R$. In this situation, $g$ is the identity map on $R$, and thus $F$ is a generalized derivation of $R$.

We conclude our paper with an application of previous results.

Theorem 18. Let $R$ be a noncommutative prime ring of characteristic different from $2, I$ a nonzero ideal of $R$, and $F$ : $R \rightarrow R$ a nonzero generalized semiderivation associated with the endomorphism $g$ and semiderivation $d$ of $R$. If $F([x, y])=$ 0 for all $x, y \in I$, then one of the following holds:

(1) $g([R, R])=(0)$, and there exists $\beta \in C$ such that $F(x)=\beta g(x)$ for all $x \in R$;

(2) $g(I)=(0)$ and $F(I)=(0)$.

Proof. In case $F$ is a generalized derivation of $R$, by [14], we have the contradiction that either $F=0$ or $R$ is commutative. Therefore, in light of Theorem 17, we assume that $F(x)=\alpha x+$ $\beta g(x)=(\alpha+\beta) x+d(x)$, for all $x \in R$. Moreover, we may consider that $\beta \neq 0$, otherwise $F([x, y])=\alpha[x, y]=0$ forces either $\alpha=0$ (i.e., $F=0$ ) or $R$, commutative, in any case a contradiction again.

By our hypothesis and for any $x, y \in I$, we have

$$
0=F([x y, y])=F([x, y] y)=g([x, y]) d(y)
$$

that is, $\alpha[x, y] d(y)=0$. Replacing $x$ by $[r, s] x$, with $r, s \in R$, it follows that $\alpha[[r, s], y] x d(y)=0$, that is, $\alpha[[r, s], y] \operatorname{Id}(y)=(0)$. Since $R$ is not commutative and using standard arguments, it follows that either $\alpha=0$ or $d(I)=(0)$.

Assume that $\alpha \neq 0$ and $d(I)=0$; then $d(R)=0$ follows from Corollary 8. Hence $g(x)=x$, for all $x \in R$, that is, $F(x)=(\alpha+\beta) x$, which means that $F$ is a generalized derivation of $R$, a contradiction.

Finally consider that $\alpha=0$ and $F(x)=\beta g(x)=\beta x+d(x)$, for all $x \in R$. Therefore $\beta g([x, y])=0$, that is, $g([x, y])=0$ which implies that $d([x, y])+\beta[x, y]=0$, for all $x, y \in I$.

If we set $D=1-g=-\beta^{-1} d$, then $D$ is a semiderivation associated with the endomorphism $g$. Moreover, $D$ satisfies the condition $D([x, y])-[x, y]=0$, for all $x, y \in I$.

Since $R$ is not commutative, by applying Theorem 14 , we conclude that either $g([R, R])=(0)$, or $g(I)=(0)$ and $D(x)=$ $x$ for all $x \in I$, that is, $d(x)=-\beta x$ for all $x \in I$, and $F(I)=$ (0).

\section{References}

[1] E. C. Posner, "Derivations in prime rings," Proceedings of the American Mathematical Society, vol. 8, pp. 1093-1100, 1957.

[2] C. Lanski, "Differential identities, Lie ideals, and Posner's theorems," Pacific Journal of Mathematics, vol. 134, no. 2, pp. 275-297, 1988.

[3] B. Hvala, "Generalized derivations in rings," Communications in Algebra, vol. 26, no. 4, pp. 1147-1166, 1998.

[4] T.-K. Lee, "Generalized derivations of left faithful rings," Communications in Algebra, vol. 27, no. 8, pp. 4057-4073, 1999.

[5] J. Bergen, "Derivations in prime rings," Canadian Mathematical Bulletin, vol. 26, no. 3, pp. 267-270, 1983.

[6] S. M. A. Zaidi, M. Ashraf, and S. Ali, "On Jordan ideals and left $(\theta, \theta)$-derivations in prime rings," International Journal of Mathematics and Mathematical Sciences, vol. 2004, no. 37, pp. 1957-1964, 2004.

[7] R. Awtar, "Lie and Jordan structure in prime rings with derivations," Proceedings of the American Mathematical Society, vol. 41, pp. 67-74, 1973.

[8] L. Oukhtite, "On Jordan ideals and derivations in rings with involution," Commentationes Mathematicae Universitatis Carolinae, vol. 51, no. 3, pp. 389-395, 2010.

[9] L. Oukhtite and A. Mamouni, "Derivations satisfying certain algebraic identities on Jordan ideals," Arabian Journal of Mathematics, vol. 1, no. 3, pp. 341-346, 2012.

[10] L. Oukhtite, "Posner's second theorem for Jordan ideals in rings with involution," Expositiones Mathematicae, vol. 29, no. 4, pp. 415-419, 2011.

[11] H. E. Bell and M. N. Daif, "On derivations and commutativity in prime rings," Acta Mathematica Hungarica, vol. 66, no. 4, pp. 337-343, 1995.

[12] J.-C. Chang, “On semiderivations of prime rings," Chinese Journal of Mathematics, vol. 12, no. 4, pp. 255-262, 1984.

[13] M. Brešar, "Centralizing mappings and derivations in prime rings," Journal of Algebra, vol. 156, no. 2, pp. 385-394, 1993.

[14] O. Gëlbashi and K. Kaya, "On Lie ideals with generalized derivations," Rossiǔskaya Akademiya Nauk, vol. 47, no. 5, pp. 1052-1057, 2006. 


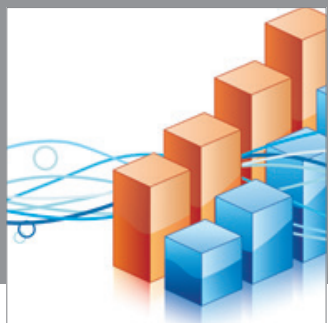

Advances in

Operations Research

mansans

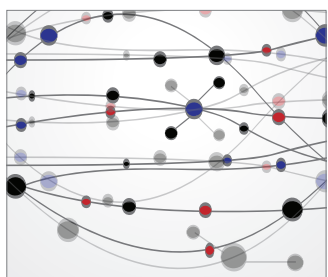

The Scientific World Journal
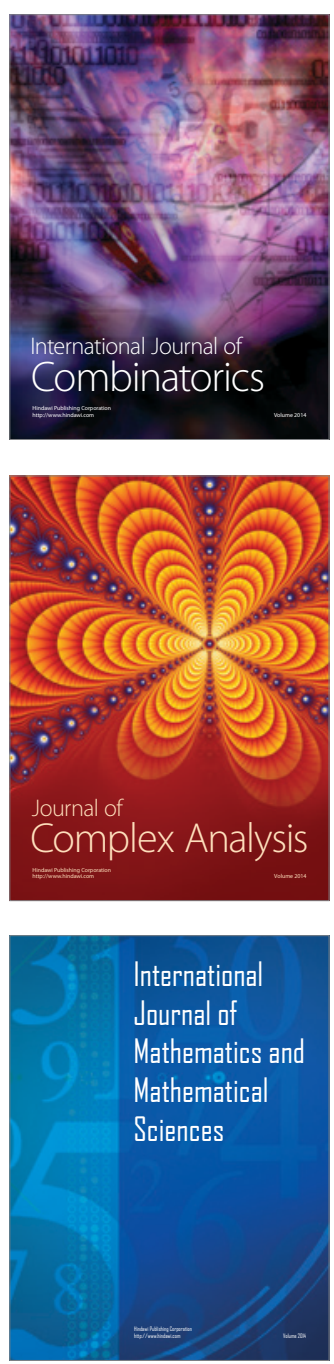
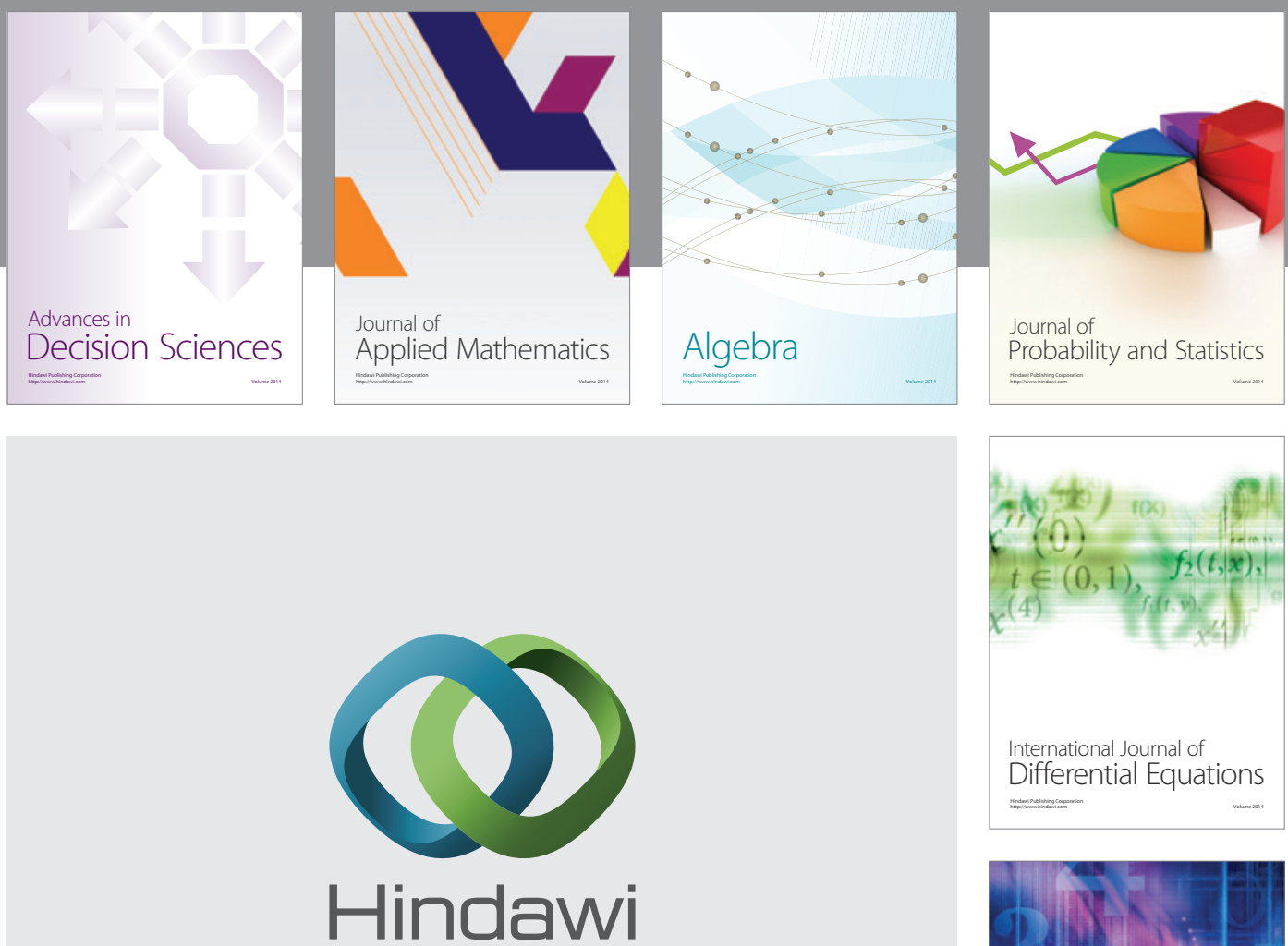

Submit your manuscripts at http://www.hindawi.com
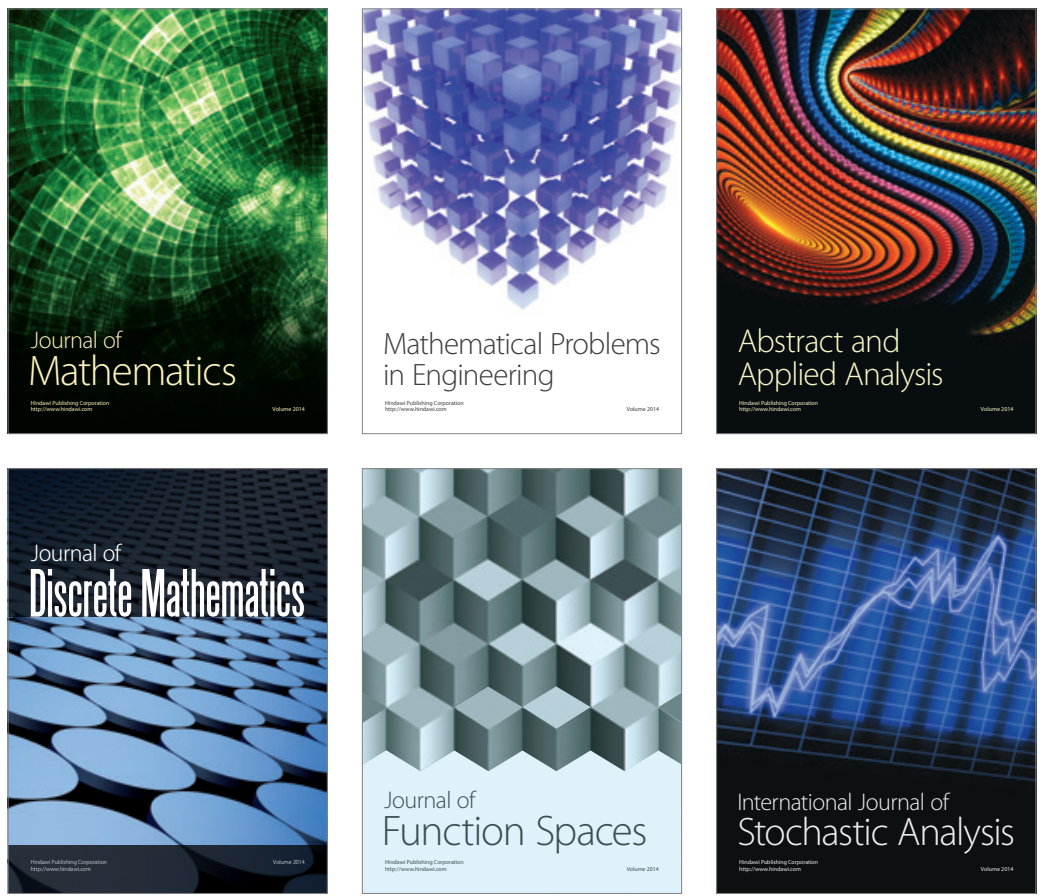

Journal of

Function Spaces

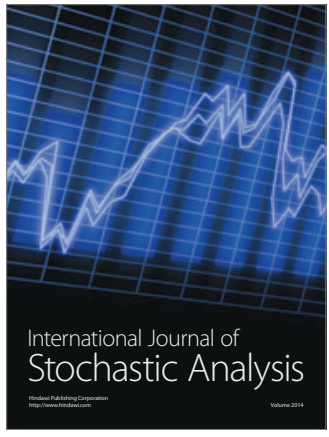

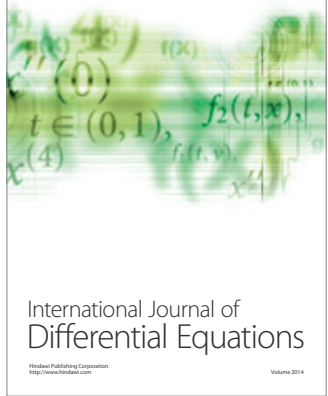
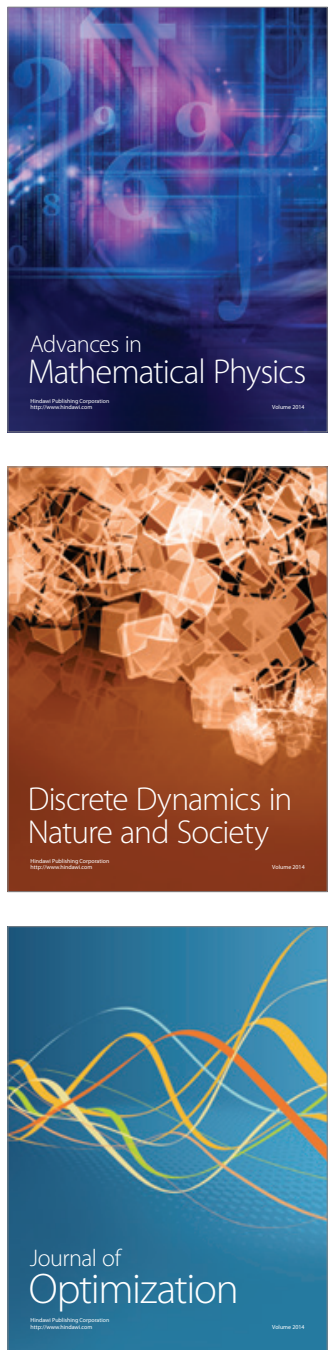